\title{
PARTICIPACIÓN POLÍTICA Y BIENESTAR SOCIAL; UN MODELO PREDICTIVO CON JÓVENES MEXICANOS
}

\author{
Edgar Alejandro Chávez Ortega y Andrés Valdez Estrella \\ FES Zaragoza, UNAM \\ México
}

\begin{abstract}
RESUMEN
La investigación tuvo como objetivo proponer un modelo predictivo para la participación política y el bienestar social con jóvenes universitarios del área metropolitana de México. Las variables predictivas de la participación política han sido estudiadas, pero las investigaciones acerca de los predictores del bienestar social son escasas; no obstante, hay evidencia de que la participación política y el bienestar social son constructos relacionados. La muestra estuvo conformada por 306 participantes ( $32.6 \%$ hombres y $67.1 \%$ mujeres) del área metropolitana. Se desarrollaron análisis de correlaciones y de regresión lineal múltiple con el método paso por paso. Se observó que la participación política se predice por las siguientes variables: eficacia política global, interés político, autoritarismo de derecha, aceptación social, actualización social y contribución social; mientras que el bienestar social se predice por los siguientes antecedentes: eficacia política global, participación política comunitaria, autoritarismo de derecha, violencia física y daño a la propiedad privada. Estos resultados corroboran investigaciones anteriores respecto a los predictores de la participación política.
\end{abstract}

\section{Palabras Clave:}

participación política, bienestar social, jóvenes, modelo predictivo

\section{Political PARTICIPATION AND SOCIAL WELFARE; A PREDICTIVE MODEL WITH YOUNG MEXICANS}

\begin{abstract}
The aim of this research was propose a predictive model for political participation and social well-being with a population of undergraduate students from the metropolitan area of Mexico. The predictive variables of political participation have been studied assiduously, but research on the predictors of social well-being is scarce, on the other hand there is evidence that political participation and social well-being can be related constructs. The sample consisted of 306 participants ( $32.6 \%$ men and $67.1 \%$ women) from the metropolitan area. Correlation and multiple linear regression analysis were performed with the step-by-step method to explore the predictive variables. Political participation was predicted by the following variables: global political efficacy, political interest, right-wing authoritarianism, social acceptance, social actualization and social contribution; while social well-being was predicted by the following antecedents: global political efficiency, community political participation, right-wing authoritarianism, physical violence and damage to private property. Regarding the predictors of political participation these results corroborate what was reported in previous research. Also, is clarified new information on the predictive variables of social well-being.
\end{abstract}

\section{Keywords:}

Political participation, social well-being, young people, predictive model

Bitácora del Artículo:

| Recibido: 19 de Marzo de 2018 | Aceptado: 1 Septiembre de 2018 | Publicado en línea: Julio-Diciembre de 2018 | 


\title{
Autoría y Derechos de Propiedad Intelectual
}

\section{PARTICIPACIÓN POLÍTICA Y BIENESTAR SOCIAL; UN MODELO PREDICTIVO CON JÓVENES MEXICANOS}

\author{
Edgar Alejandro Chávez Ortega y Andrés Valdez Estrella \\ FES Zaragoza, UNAM \\ México
}

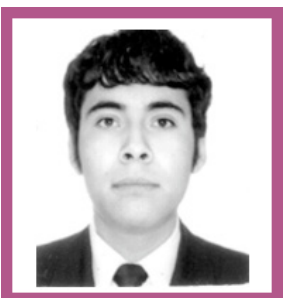

Edgar Alejandro Chávez Ortega

FES Zaragoza, UNAM

Correo: alex_eaco1970@hotmail.com

Licenciado en Psicología por la UNAM-FES Zaragoza. Ponente de trabajos de investigación en congresos nacionales e internacionales; participante en proyectos de investigación financiados por el PAPIME. Actualmente colaborador en el Departamento de Educación Continua de la FES Zaragoza.

Ver más...

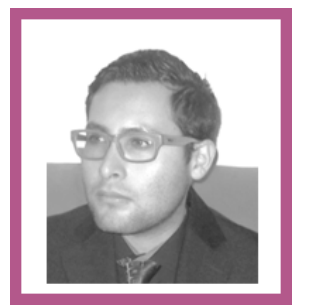

Andrés Valdez Estrella FES Zaragoza, UNAM

Correo: andres.valdez.est@gmail.com

Licenciado en Psicología por la UNAM-FES Zaragoza. Actualmente investiga los temas de liderazgo y personalidad.

Ver más...

\section{Contribución de los Autores}

Edgar Chávez participó en la redacción de todo el manuscrito, y en especial la elaboración de marco teórico y conclusiones; también realiza trabajo de campo al aplicar los instrumentos y colaborar en el análisis estadístico. | Andrés Valdez tiene una destacada participación en la elaboración de la metodología, el desarrollo de análisis estadísticos, incluyendo la redacción de resultado y discusiones. Así como su colaboración en el marco teórico y en el trabajo completo.

\section{AGRADECIMIENTOS}

A la Dra. Fabiola Itzel Villa-George y al Dr. Pedro Wolfgang Velasco Matus. Los estudiantes que colaboraron con su participación.

\section{DATOS DE FILIACIÓN DE LOS AUtORES}

Facultad de Estudios Superiores Zaragoza, UNAM

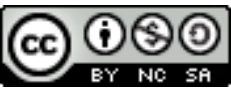

Copyright: (c) 2018 Chavéz-ortega, E. A. \& Valdez-Estrella, A.

Este es un artículo de acceso abierto distribuido bajo los términos de la licencia Creative Commons Reconocimiento-NoComercial 4.0 Internacional, por lo que su contenido gráfico y escrito se puede compartir, copiar y redistribuir total o parcialmente sin necesidad de permiso expreso de sus autores con la única condición de que no se puede usar con fines directamente comerciales y los términos legales de cualquier trabajo derivado deben ser los mismos que se expresan en la presente declaración. La única condición es que se cite la fuente con referencia a la Revista Digital Internacional de Psicología y Ciencia Social y a sus autores. 


\section{TABLA DE CONTENIDO}

Participantes, 26

La muestra, 26

El cuestionario, 27

Procedimiento, 27

Mediciones, 28

Análisis estadísticos, 28

HaLLAZgos

Análisis de las reglas mencionadas de modo espontáneo por campo de conocimiento, 29 


\section{INTRODUCCIÓN}

$\mathrm{E}$ 4 de julio de $2012^{1}$ se efectuó en México el proceso electoral para elegir de Presidente de la República y de otros funcionarios públicos. En dicho proceso electoral se registró una participación por parte de la ciudadanía de " $62.08 \%$, lo que representa un incremento respecto a lo observado en 2006, con 58.55\%; sin embargo, este porcentaje es inferior a los niveles registrados en las votaciones de 1994 (77.16\%)" (Instituto Federal Electoral, 2013). ¿A qué se debe la participación política? O, ¿a qué se debe la falta de participación política? El estudio de Rodríguez (2004) muestra que para los ciudadanos la participación política es votar, que asocian participación política y gobierno, y éste a su vez lo relacionan con la corrupción. Los mexicanos no confían en ninguna institución política, y pocas veces recurren a ellas para hacer cambios políticos; por tal motivo, la participación electoral de los ciudadanos no es muy alta.

Algunas personas se interesan más en la política. No se sabe con exactitud a qué se debe esto. El interés en la política es un predictor de los comportamientos políticos que hacen funcionar a la democracia. Las personas más interesadas en la política suelen ser personas que voten y que también participen en la política de otros modos (Córdova, Alejandre, Vázquez del Mercado, 2015). "La participación política describe actividades directas o indirectas que realizan los ciudadanos para influir en las decisiones o en la elección de los gobernantes, e incluye formas convencionales (participación electoral, activismo partidario) y no convencionales (actividades de protesta, contacto con autoridades)" (Várela, Loreto y Cumsille, 2015, p. 732).

Delfino, Zubieta y Muratori (2010) reconocen una primera clasificación que divide la participación en dos: convencional y no convencional. La primera modalidad (convencional) está caracterizada sobre todo por los actos o acciones relacionados indirecta o directamente con los procesos electorales; ejemplo de estas acciones son: actividades de campañas políticas, actividades comunitarias, contactos con la administración y el voto. La segunda modalidad (no convencional) se observa como una participación heterogénea donde su campo de acción está entre lo legal y lo ilegal; en esta modalidad se identifican actos como boicots, manifestaciones, desobediencia civil, etcétera.

1 Este trabajo fue elaborado antes del proceso electoral del 2018
Sin embargo, los autores expresan diferentes inquietudes acerca de una sola clasificación de la participación política, por lo cual optan por ampliar la clasificación en cuatro modalidades de participación: 1) persuasión electoral, que incluye actividades como convencer a otros para que voten como uno y acudir a mítines; 2) participación convencional, que se caracteriza por las actividades que se mantienen en la legalidad vigente y que tratan de incidir en el curso de los acontecimientos político-sociales (como votar, enviar escritos a la prensa, manifestaciones autorizadas y huelgas autorizadas); 3) participación violenta, que integra acciones como daños a la propiedad y violencia armada, y 4) participación directa pacífica, que incluye actividades que, si bien pueden desbordar el marco de la legalidad establecida, no son necesariamente violentas (como ocupación de edificios, boicots, cortes de tráfico, manifestaciones no autorizadas y huelgas no autorizadas) (Delfino, Zubieta \& Muratori, 2013, p. 307).

En otras investigaciones, como la de Murga (2009), retomando la postura de Durand, se estiman cuatro modalidades de participación: 1) militante; se denomina al sujeto que une a su interés subjetivo por la política las acciones destinadas a lograr los intereses de la organización o partido con el cual se identifica; 2) movilizado, donde la persona, aunque participa, no tiene interés en la política; entonces su participación está dirigida a sus propios intereses; 3) pasivo, la persona interviene de manera subjetiva, pero se abstienen de actuar, y 4) apolítico, donde no se participa ni existe interés en la política.

La participación política (PP), aunque no es concluyente, parece estar relacionada con el bienestar individual o con el bienestar subjetivo. En la investigación de Temkin y Flores-Ivich (2017), los resultados obtenidos permiten entender que las personas más satisfechas con su vida son las que participan, y en específico la participación no violenta se relaciona con un bienestar subjetivo. Los autores determinan que esto ocurre porque la participación desencadena procesos psicológicos de autoeficacia y utilidad procedimental, que a su vez generan mayor satisfacción.

De este modo la participación, desde una orientación sociopsicológica de Sorribas y Brussino (2017), se da de modo multidimensional, por lo cual son necesarias diferentes variables sociopsicológicas para distinguirla. Dichas variables son:

1) Eficacia política. Es considerada como la creencia subjetiva de un individuo, o grupo, de tener capacidades para participar e influir en el curso de los sucesos políticos. 
2) Interés político, Se trata de una sensación de curiosidad por los asuntos políticos.

3) Conocimiento político. Puede ser definido como el nivel de entendimiento que tienen los ciudadanos del funcionamiento político en el cual están inmersos.

4) Confianza política. Se define como la confianza que tienen los ciudadanos en las acciones y la gente que representa al gobierno.

5) Individualismo y colectivismo (I/C). Tienen dos dimensiones; el individualismo cultural se caracteriza por la autonomía intelectual, mientras que el colectivismo cultural se apega a los valores de jerarquía y arraigo; el individualismo político se orienta a motivaciones egoístas propias de la política institucionalizada, y el colectivismo político se enfoca en acciones que pretenden el bienestar común, propias de la PP comunitaria.

6) Actitudes ideológicas. Son pauta de evaluación cognoscitivas que median en algún grado el comportamiento de los individuos; surgen determinados por relaciones familiares, disposiciones de la personalidad, perspectivas del mundo y objetivos motivacionales; además tienden a ubicarse en el continuo de pensamiento político de izquierda y derecha (Weber y Federico, 2007).

\section{Participación política en jóvenes universitarios}

En el mismo proceso electoral de 2012 en México, se observó que los niveles más altos de participación son en la población de 40 a 79 años de edad. De este modo se detecta que la menor participación es en los adultos mayores de 80 años y en los jóvenes (20 a 39 años). Sin embargo, los jóvenes de 18 o 19 años que votan por primera vez son una excepción al lograr una participación de 63\% (Leyva, Muñiz \& Flores, 2016).

Los jóvenes universitarios son una cultura particular juvenil donde el contexto escolar, el distanciamiento entre el mundo juvenil y adulto, la condición de pares y la exigencia académica son algunas características de la cultura universitaria (Arias-Cardona \& Alvarado, 2015). Se cree que un alto nivel educativo favorece la acción política al romper barreras cognitivas, fomentar la interacción y la disposición de información política. Sin embargo, los resultados aportados por Murga (2009) muestran que no hay relación entre el nivel educativo y la participación política.

\section{Bienestar social}

La Organización Mundial de la Salud (1998) define la salud como "Un estado de completo bienestar físico, social y mental, no solamente la ausencia de enfermedad y do- lencias". Desde la psicología el abordaje de estos constructos se divide en tres tradiciones distintas; la primera es conocida como hedónica, propia del bienestar subjetivo (Diener, Suh, Lucas \& Smith, 1999; Velasco, 2015); la segunda, denominada eudaimónica, representada por el bienestar psicológico (Ryff \& Keyes, 1995), y la tercera se enfoca en evaluar el bienestar social (Keyes, 1998; Keyes \& Shapiro, 2004; Zubieta \& Delfino, 2010).

Acorde con lo expuesto por Cárdenas (2011), el estudio del bienestar adquiere importancia social si se considera el contexto político, social y económico que se vive en México; el conjunto de circunstancias que condicionan la vida de los ciudadanos mexicanos, en particular los procesos económicos propiciados por la globalización, ponen en riesgo el bienestar social de los individuos que conforman esta sociedad. El enfoque psicométrico de Keyes (1998) sería el más adecuado para calcular esta variable, a pesar de las debilidades intrínsecas de este modelo de evaluación del bienestar social.

El constructo de bienestar social expresa con precisión el ajuste óptimo entre las personas y su contexto. Entre las variables que promueven el bienestar psicológico destacan la participación social en grupos formales o informales, como la participación política. Sin embargo, la función que desempeña la realización de actividades grupales para mantener y promover el bienestar psicológico aún no se ha investigado de manera exhaustiva. También hay variables individuales que pueden predecir de manera consistente el bienestar social como el locus de control. En este trabajo, una de las variables personales que podría predecir de modo certero el bienestar social es la eficacia política global (Rollero, 2013).

Son y Wilson (2012) hicieron un estudio con una muestra de ciudadanos estadounidenses; se corroboró que las actividades de voluntariado aumentan el bienestar eudaimónico y el bienestar social; de modo inverso las personas con un mayor grado de bienestar eudaimónico, hedónico y social tenían mayores probabilidades de involucrarse en actividades de voluntariado. Esta información indica que las actividades sociales que se desarrollan para resguardar la integridad de otras personas, como la participación política, pueden tener un impacto positivo en el bienestar social.

En esta investigación se considera al bienestar social como un constructo conformado por cinco factores: 1) integración social; evalúa la pertenencia social, como los lazos con la familia, vecinos o amigos; hace referencia al proceso de aceptación que se efectúa al comparar los valores o creencias individuales con su contraparte social; 2) aceptación social; atribución positiva que se hace en las personas del entorno; 3) contribución social; 
percepción de utilidad dentro de la sociedad; se asemeja al concepto de autoeficacia porque es la capacidad que percibe el individuo para colaborar mediante sus acciones con el desarrollo colectivo; 4) actualización social; percepción positiva del desarrollo social dirigido a promover el bienestar; este componente se enfoca a evaluar la creencia de que existe una clase de progreso social, y 5) coherencia social; preocupación por entender lo que sucede en el mundo y creencia de que se entiende lo que acontece alrededor; es el equivalente social de considerar nuestra vida personal como coherente y relevante (Blanco y Díaz, 2005; Keyes, 1998; Keyes \& Shapiro, 2004).

Blanco y Díaz (2005) hicieron una investigación para evaluar las propiedades psicométricas del instrumento de Keyes (1998) de bienestar social con 469 personas, 192 estudiantes de la Facultad de Psicología de la Universidad Autónoma de Madrid y 277 trabajadores de diversas empresas de la Comunidad de Madrid. Sus resultados indican índices aceptables de consistencia interna con valores Alfa de Cronbach, que oscilaron entre 0.68 y 0.83 . Mediante análisis factorial confirmatorio determinaron que el modelo que mejor ajusta para las escalas de bienestar social se conforma por cinco factores de primer orden, acorde con los cinco constructos ya descritos.

En un estudio hecho por Coppari, Aponte, Ayala, Moreno, Quevedo, Sola y Velázquez (2013) en una comunidad rural de Paraguay con una muestra de 113 participantes se determinó que la PP agrupada como un constructo unitario y el bienestar social (BS) global tienen una correlación bilateral de Pearson positiva y significativa $(r=0.981, p<0.001)$. Estos resultados indican una estrecha relación entre las acciones políticas y el sentimiento de bienestar que se relaciona con sentirse parte de la comunidad y capaz de hacer una contribución social valiosa. De manera consecuente, en la presente investigación se espera encontrar una estrecha relación entre dos constructos con una muestra de adultos jóvenes que viven en un entorno urbano.

Acorde con lo argumentado por Temkin y Flores-Ivich (2017), las personas están interesadas en evaluar el proceso de toma de decisiones políticas que se establecen a partir de la democracia; participar en procesos políticos puede aumentar la felicidad de las personas, por lo que son más propensas a evaluar las cuestiones relacionadas con el bienestar social de una manera más positiva. La participación política que ejercen las personas permite aumentar su sentido de integración social, y mediante ella se pretende alcanzar alguna forma de bienestar.

\section{Variables antecedentes de la participación política y el bienestar social}

El interés político es una de las variables más relevantes para predecir el conocimiento político (Brussino, Medrano, Sorribas, \& Rabbia, 2011). El interés político podría relacionarse con el bienestar social porque expresa el interés que tiene una persona por conocer la realidad que lo rodea en términos de acontecimientos políticos.

El autoritarismo de derecha (AD) se compone por tres actitudes sociales: el convencionalismo, la sumisión a la autoridad y la agresión en contra de grupos exteriores. La combinación de estas tres actitudes genera una dimensión actitudinal unitaria. Este constructo es relevante para explicar variables de la psicología política (como el prejuicio, la intolerancia, el nacionalismo, la participación, las preferencias políticas y las elecciones electorales). EI AD también se relaciona de manera estrecha con la "cultura de la guerra" (Weber \& Federico, 2007).

La eficacia política es una de las variables con mayor relación empírica respecto a la participación política. La variable es definida como "la creencia subjetiva de un individuo o grupo de poseer capacidades para participar e influir en el curso de los sucesos políticos" (Brussino, Rabbia y Sorribas, 2009, p. 281). En esta investigación se evalúa la eficacia política global conceptualizada como las creencias dedicadas a valorar las competencias de entendimiento político y capacidad de participación política de los ciudadanos (Niemi, Craig \& Mattei, 1991).

Laca-Arocena, Mejía-Ceballos y Yañez-Velasco (2010) hicieron un estudio transversal con una muestra mexicana de Colima con 211 participantes; en este estudio se corrobora que el bienestar social correlaciona de manera positiva débil o moderada y significativa con el interés político; en particular con las subescalas de aceptación social, actualización social y coherencia social.

En un estudio desarrollado por Laca-Arocena, Santana-Aguilar, Ochoa-Madrigal y Mejía-Ceballos (2011) con muestras de siete estados de México (Campeche, Colima, Ciudad de México, Nayarit, Nuevo León, Puebla y Veracruz) con 568 participantes se encontró que el interés político correlaciona débil y moderadamente de manera positiva y significativa con las subescalas que conforman el bienestar social como lo conceptualiza Keyes (1998).

Con la revisión de la literatura se plantea la siguiente pregunta de investigación. ¿Cuáles son las variables 
que pueden predecir la relación entre el bienestar social y participación política en jóvenes universitarios?

La presente investigación estableció como objetivo proponer un modelo predictivo para la participación política y el bienestar social con una población de jóvenes universitarios del área metropolitana de México.

\section{MÉTOdo}

\section{Participantes}

Se trabajó con una muestra no probabilística intencional de 307 participantes; 32.6\% (100) hombres y $67.1 \%$ (206) mujeres de la Ciudad de México y el área metropolitana, con un rango de edad entre 17-34 años $(M=$ 20.69 DE =1.7). El nivel educativo de los participantes era de licenciatura con $96.4 \%$ (296) provenientes de un sistema educativo público, y $98.7 \%$ (303) de ellos no eran militantes de algún partido político. Los criterios de inclusión eran estar actualmente inscritos en el sistema educativo superior.

\section{Procedimiento}

Se explicó a los participantes que su colaboración sería para apoyar una investigación que se desarrollaba en la Facultad de Estudios Superiores Zaragoza, en el área de Psicología; asimismo se les informó que todos y cada uno de los datos que proporcionaran serían tratados de manera confidencial y con absoluto profesionalismo, por lo cual se les solicitaba que respondieran de la manera más honesta posible y firmaran hoja de consentimiento informado. La recolección de datos se hizo entre los meses de octubre y noviembre de 2017.

\section{Mediciones}

Hoja de datos sociodemográficos. Diseñada ex profeso para conocer características relevantes de los participantes; los datos recolectados con este medio se encuentran en la sección Participantes.

Escala de participación política. Diseñada por García y Barragan (2008), compuesta por 15 ítems que integran tres subescalas: 1) participación política comunitaria (7 ítems; por ejemplo, "Juntado firmas o firmar desplegados, cartas o peticiones"); 2) desobediencia civil no violenta (4 ítems; como "Manifestaciones dentro de la ley como marchas"), y 3) violencia física y daño a la propiedad privada (3 ítems; como "Dañado propiedad privada [autos, comercios, etcétera]"). Por último, en esta escala hay un reactivo único dedicado a evaluar la disposición a votar que tienen las personas . Votar es una manera única de conducta política separada de las subescalas mencionadas. Estos ítems están organizados en escala tipo Likert de seis puntos, que varía de $1=$ No participaría a $6=$ He participado.

Escala de bienestar social. Diseñada por Keyes (1998) y adaptada al castellano por Blanco y Díaz (2005), compuesta por 25 ítems que integran cinco subescalas: 1) integración social ( 5 ítems, como "Siento que soy una parte importante de mi comunidad"); 2) aceptación social (6 ítems, como "Creo que la gente no es de fiar"); 3) contribución social (5 ítems, como "Creo que puedo aportar algo al mundo"); 4) actualización social (5 ítems, como "Para mí el progreso social es algo que no existe"), y 5) coherencia social (4 ítems, como "No entiendo lo que está pasando en el mundo"). Estos ítems están organizados en escala tipo Likert de cinco puntos, que varía de $1=$ Fuertemente en desacuerdo a 5 = Fuertemente de acuerdo.

Escala de interés político. Diseñada por Brussino, Medrano, Sorribas y Rabbia (2011), está compuesta por seis ítems (como "Disfruto las conversaciones sobre temas políticos y gubernamentales") organizados en escala tipo Likert de cinco puntos, que varía de $1=$ Fuertemente en desacuerdo a $5=$ Fuertemente de acuerdo.

Escala de autoritarismo de derecha. Diseñada por Weber y Federico (2007), está compuesta por 12 ítems (como "La gente debe prestar menor atención a la biblia y a otras viejas tradiciones de origen religioso y en su lugar desarrollar sus estándares personales de lo que es moral e inmoral") organizados en escala tipo Likert de seis puntos, que varía de 1 = Fuertemente en desacuerdo a 6 = Fuertemente de acuerdo.

Escala de eficacia política global. Diseñada por Niemi, Craig y Mattei (1991) y adaptada por Brussino, Medrano, Sorribas y Rabbia (2011), está compuesta por ocho ítems (como "La opinión de gente como usted ejerce alguna influencia sobre las decisiones del Gobierno") organizados en escala tipo Likert de seis puntos, que varía de $1=$ Totalmente en desacuerdo a $6=$ Totalmente de acuerdo.

\section{Análisis estadísticos}

Se hicieron análisis de regresión lineal múltiple con el método paso por paso para explorar las variables predictivas de la PP y el BS; esta metodología no indica causalidad entre las variables (Hayes, 2013); los análisis se estudiaron por medio del programa SSPS V. 20.

\section{Resultados}

La confiabilidad de las subescalas de PP oscilan entre a $=0.62$ y $\alpha=0.84$, mientras que la confiabilidad de las subescalas de BS oscilan entre $\alpha=0.60$ y $\alpha=0.80$; por 
otro lado, la escala de interés político tiene alta confiabilidad $(\alpha=0.92)$; la escala de autoritarismo de derecha tiene un alfa de Cronbach de 0.53, y la escala de eficacia política global tiene un alfa de Cronbach de 0.64. Las confiabilidades que oscilan entre $\alpha=0.60$ podrían parecer deficientes; sin embargo, acorde con Argibay (2006) para los instrumentos conformados con 10 ítems, es aceptable obtener una confiabilidad aproximada de $\alpha=0.60$.

A partir de la tabla de correlaciones (tabla 1) fue posible observar que la relación entre PP y BS es más intensa entre las subescalas de participación política comunitaria y contribución social $(r=0.312, p<0.01)$, actualización social $(r=0.269, \mathrm{p}<0.01)$ y coherencia social $(r=0.259, p<0.01)$. Además se encontraron relaciones positivas y significativas entre participación política comunitaria e interés políticos $(r=0.459, p<0.01)$, al igual que la desobediencia civil no violenta y el interés político $(r=0.450, p<0.01)$. Se obtuvo una correlación negativa y significativa entre la desobediencia civil no violenta y las creencias propias del autoritarismo de derecha $(r=-0.361, p<0.01)$. Por último, se observaron correlaciones positivas y significativas entre la participación política comunitaria y la eficacia política global

Tabla 1.

Correlaciones, medias, desviación estándar y confiabilidad de las escalas

\begin{tabular}{|c|c|c|c|c|c|c|c|c|c|c|c|c|c|c|c|c|}
\hline Escala & $\begin{array}{c}\text { No. } \\
\text { DE } \\
\text { ITEMS }\end{array}$ & $M$ & DE & $\alpha$ & 1 & 2 & 3 & 4 & 5 & 6 & 7 & 8 & 9 & 10 & 11 & 12 \\
\hline 1. V & 1 & 4.99 & 1.45 & - & - & $.243^{* *}$ & .111 & -.034 & $.154^{* *}$ & .029 & $.115^{*}$ & .105 & $.143^{*}$ & .070 & -.066 & $.182^{* *}$ \\
\hline 3. DCNV & 4 & 2.67 & 1.33 & .80 & & & - & $.328^{* *}$ & $.147^{*}$ & $.141^{*}$ & $.146^{*}$ & $.179 * *$ & $.131^{*}$ & $.450^{* *}$ & $-.361^{* *}$ & $.284^{* *}$ \\
\hline 4. VFDPP & 3 & 1.32 & .63 & .62 & & & & - & -.061 & -.044 & $-.129^{*}$ & -.094 & -.004 & $.156^{* *}$ & -.088 & .112 \\
\hline 7. CS & 5 & 3.95 & .69 & .76 & & & & & & & - & $.323^{* *}$ & $.415^{* *}$ & $.255^{* *}$ & $-.287^{* *}$ & $.333^{* *}$ \\
\hline 8. ACTS & 5 & 3.31 & .58 & .60 & & & & & & & & - & $.382^{* *}$ & $.155^{* *}$ & -.066 & $.202^{* *}$ \\
\hline 9.COHS & 4 & 3.77 & .74 & .71 & & & & & & & & & - & $.174^{* *}$ & $-.150 * *$ & $.289 * *$ \\
\hline 10. IP & 6 & 2.87 & .96 & .92 & & & & & & & & & & - & $-.248^{* *}$ & $.525^{* *}$ \\
\hline
\end{tabular}

$(r=0.390, p<0.01)$, y el interés político y la eficacia política global $(r=0.525, p<0.01)$.

Se efectuaron análisis de regresión lineal múltiple con el método paso por paso para explorar las variables predictivas de la PP y el BS; esta metodología no indica causalidad entre las variables (Hayes, 2013), por lo que se propone una contextualización causal recíproca entre la PP y el BS (Salanova, Llorens \& Schaufeli, 2011; Son \& Wilson, 2012). Se evaluó que las variables cumplieran los criterios de independencia de los errores o residuos, homocedasticidad, normalidad, linealidad y no colinealidad. Sólo se observó que las variables de voto y violencia física y daño de la propiedad privada incumplen el supuesto de normalidad de distribución de los residuos (Moreno, 2008).

En la tabla 2 se puede corroborar que la única variable antecedente con valor significativo para predecir la conduc- ta de voto es la eficacia política global, explicando un porcentaje de la varianza de la variable consecuente de $2.9 \%$, lo que indica un modelo predictivo de eficacia baja.

Tabla 2.

Resumen del análisis de regresión paso a paso para los predictores del voto

\begin{tabular}{|c|c|c|c|c|c|}
\hline \multirow{2}{*}{ VARIABLES } & \multirow[t]{2}{*}{ B } & \multirow{2}{*}{ EE } & \multirow[t]{2}{*}{ B } & \multicolumn{2}{|c|}{$\begin{array}{l}\text { INTERVALO DE } \\
\text { CONFIANZA }\end{array}$} \\
\hline & & & & Mínimo & MÁxIMo \\
\hline $\begin{array}{l}\text { 1er Paso } \\
\text { Eficacia } \\
\text { política } \\
\text { global }\end{array}$ & .298 & .106 & .171 & .090 & .506 \\
\hline
\end{tabular}


En la tabla 3 se muestra que la variable antecedente (participación política comunitaria) puede ser predicha por cinco variables; en el primer paso se ubica el interés político explicando $22.9 \%$ de la varianza; en el segundo paso se encontró un predictor negativo: el autoritarismo de derecha, explicando $27.7 \%$ de la varianza; en el tercer paso se incorpora la aceptación social explicando $31.6 \%$ de la varianza; en el cuarto paso se integra la eficacia política global, explicando $33.2 \%$ de varianza, y en el quinto paso se incorpora la actualización social explicando un índice de varianza de $34.3 \%$ de la variable consecuente; los resultados muestran un modelo predictivo de eficacia media.

Tabla 3.

Resumen del análisis de regresión paso a paso para los predictores de participación política comunitaria

\begin{tabular}{|c|c|c|c|c|c|}
\hline \multirow[t]{2}{*}{ VARIABLES } & \multirow[t]{2}{*}{$\mathbf{B}$} & \multirow[t]{2}{*}{ EE } & \multirow[t]{2}{*}{ B } & \multicolumn{2}{|c|}{$\begin{array}{l}\text { INTERVALO DE } \\
\text { CONFIANZA }\end{array}$} \\
\hline & & & & Mínimo & MÁxıMO \\
\hline $\begin{array}{l}\text { 1er Paso } \\
\text { Interés } \\
\text { político }\end{array}$ & .374 & .070 & .320 & .235 & .513 \\
\hline $\begin{array}{l}2^{\circ} \text { Paso } \\
\text { Autoritarismo } \\
\text { de derecha }\end{array}$ & -.392 & .097 & -.213 & -.584 & -.200 \\
\hline $\begin{array}{l}\text { 3er Paso } \\
\text { Aceptación } \\
\text { social }\end{array}$ & .234 & .092 & .139 & .052 & .416 \\
\hline $\begin{array}{l}4^{\circ} \text { Paso } \\
\text { Eficacia } \\
\text { política global }\end{array}$ & .185 & .082 & .135 & .023 & .346 \\
\hline $\begin{array}{l}5^{\circ} \text { Paso } \\
\text { Actualización } \\
\text { social }\end{array}$ & .221 & .106 & .117 & .013 & .429 \\
\hline $\begin{array}{l}\text { Nota: } \mathrm{R}^{2}=0.229 \\
<0.001), \mathrm{R}^{2}=0 . \\
4(p<0.05), \mathrm{R}^{2}=\end{array}$ & $\begin{array}{l}\text { a el } p \\
\text { para } \\
343 p\end{array}$ & $\begin{array}{l}1(p< \\
\text { aso } 3\end{array}$ & $\begin{array}{l}01), R^{2} \\
0.001 \\
p<0 .\end{array}$ & $\begin{array}{l}277 \text { para } \\
{ }^{2}=0.332 p\end{array}$ & $\begin{array}{l}\text { paso } 2 \text { ( } p \\
\text { ira el paso }\end{array}$ \\
\hline
\end{tabular}

En la tabla 4 se muestran los antecedentes predictivos de desobediencia civil no violenta en dos pasos; en el primer paso está el interés político explicando $22.4 \%$ de la varianza; en el segundo paso se incorpora el autoritarismo de derecha como predictor negativo, explicando un índice de varianza de $28.4 \%$ de la variable consecuente; los resultados indican un modelo predictivo de eficacia media.

En la tabla 5 se muestran los antecedentes predictivos de la violencia física y el daño a la propiedad privada; en el primer paso está el interés político explicando $1.9 \%$ de la varianza; en el segundo paso se incorpora la contribución social como un predictor negativo, explicando $4.7 \%$ de la varianza; los resultados indican un modelo predictivo de eficacia baja.

\section{Tabla 4.}

Resumen del análisis de regresión paso a paso para los predictores de desobediencia civil no violenta

\begin{tabular}{l|l|l|l|l|l|}
\multicolumn{1}{|c}{ VARIABLES } & B & \multicolumn{5}{c}{$\begin{array}{c}\text { INTERVALO DE } \\
\text { CONFIANZA }\end{array}$} \\
$\begin{array}{l}\text { Ier Paso } \\
\begin{array}{l}\text { Interés } \\
\text { político }\end{array}\end{array}$ & .559 & .077 & .394 & .407 & .711 \\
\hline $\begin{array}{l}2^{\circ} \text { Paso } \\
\text { Autoritarismo } \\
\text { de derecha }\end{array}$ & -.574 & .121 & -.259 & -.812 & -.336 \\
\hline $\begin{array}{l}\text { Nota: } R^{2}=0.224 \text { para el paso } 1(p<0.001), R^{2}=0.284 \text { para el paso } 2(p \\
<0.001) .\end{array}$ \\
\hline
\end{tabular}

\section{Tabla 5.}

Resumen del análisis de regresión paso a paso para los predictores de violencia física y daño de la propiedad privada

\begin{tabular}{|c|c|c|c|c|c|}
\hline \multirow{2}{*}{ VARIABLES } & \multirow[t]{2}{*}{ B } & \multirow[t]{2}{*}{ EE } & \multirow[t]{2}{*}{ B } & \multicolumn{2}{|c|}{$\begin{array}{l}\text { INTERVALO DE } \\
\text { CONFIANZA }\end{array}$} \\
\hline & & & & Mínimo & MÁxIMO \\
\hline $\begin{array}{l}\text { 1er Paso } \\
\text { Interés } \\
\text { político }\end{array}$ & .126 & .043 & .181 & .041 & .211 \\
\hline $\begin{array}{l}2^{\circ} \text { Paso } \\
\text { Autoritarismo } \\
\text { de derecha }\end{array}$ & -.164 & .059 & -.173 & -.280 & -.049 \\
\hline $\begin{array}{l}\text { Nota: } R^{2}=0.019 \\
<0.001)\end{array}$ & & $1(p$ & 5), $R^{2}$ & 047 para & paso 2 ( $p$ \\
\hline
\end{tabular}

En la tabla 6 se corrobora que la única variable antecedente con valor significativo para predecir la integración social es la eficacia política global, explicando un índice de la variable consecuente de $9.5 \%$, lo que indica un modelo predictivo de eficacia baja.

\section{Tabla 6.}

Resumen del análisis de regresión paso a paso para el predictor de integración social

\begin{tabular}{|c|c|c|c|c|c|}
\hline \multirow[t]{2}{*}{ VARIABLES } & \multirow[t]{2}{*}{ B } & \multirow[t]{2}{*}{ EE } & \multirow[t]{2}{*}{ B } & \multicolumn{2}{|c|}{$\begin{array}{l}\text { INTERVALO DE } \\
\text { CONFIANZA }\end{array}$} \\
\hline & & & & Mínimo & MÁxIMO \\
\hline $\begin{array}{l}\text { 1er Paso } \\
\text { Interés } \\
\text { político }\end{array}$ & .249 & .047 & .309 & .157 & .342 \\
\hline
\end{tabular}

En la tabla 7 se observa que la única variable antecedente con valor significativo para predecir la aceptación social es la participación política comunitaria, ex- 
plicando un índice de la variable consecuente de 5.5\%, lo cual indica un modelo predictivo de eficacia baja.

Tabla 7.

Resumen del análisis de regresión paso a paso para el predictor de aceptación social

\begin{tabular}{|c|c|c|c|c|c|}
\hline \multirow[t]{2}{*}{ VARIABLES } & \multirow[t]{2}{*}{ B } & \multirow[t]{2}{*}{ EE } & \multirow[t]{2}{*}{ B } & \multicolumn{2}{|c|}{$\begin{array}{l}\text { INTERVALO DE } \\
\text { CONFIANZA }\end{array}$} \\
\hline & & & & Mínimo & MÁxIMO \\
\hline $\begin{array}{l}\text { 1er Paso } \\
\text { Interés } \\
\text { político }\end{array}$ & .139 & .036 & .234 & .069 & .210 \\
\hline
\end{tabular}

En la tabla 8 se localizan los antecedentes predictivos de la contribución social; en el primer paso está la eficacia política global, explicando $11.8 \%$ de la varianza; en el segundo paso se incorpora el autoritarismo de derecha como predictor negativo, explicando $17.4 \%$ de la varianza; en el tercer paso se ubicó la violencia física y daño a la propiedad privada como predictor negativo, explicando $19.3 \%$ de la varianza; en el cuarto paso se integra la participación política comunitaria, explicando $21 \%$ de la varianza.

\section{Tabla 8.}

Resumen del análisis de regresión paso a paso para los predictores de contribución social

\begin{tabular}{|c|c|c|c|c|c|}
\hline \multirow{2}{*}{ VARIABLES } & \multirow[t]{2}{*}{ B } & \multirow{2}{*}{ EE } & \multirow[t]{2}{*}{ ß } & \multicolumn{2}{|c|}{$\begin{array}{l}\text { INTERVALO DE } \\
\text { CONFIANZA }\end{array}$} \\
\hline & & & & Mínimo & MÁxIMo \\
\hline $\begin{array}{l}\text { 1er Paso } \\
\text { Interés } \\
\text { político }\end{array}$ & .223 & .052 & .258 & .121 & .326 \\
\hline $\begin{array}{l}2^{\circ} \text { Paso } \\
\text { Autoritarismo } \\
\text { de derecha }\end{array}$ & -.246 & .067 & -.214 & -.379 & -.114 \\
\hline $\begin{array}{l}\text { 3er Paso } \\
\text { Aceptación } \\
\text { social }\end{array}$ & -.168 & .060 & -.154 & -.287 & -.049 \\
\hline $\begin{array}{l}4^{\circ} \text { Paso } \\
\text { Eficacia } \\
\text { política global }\end{array}$ & .091 & .039 & .147 & .015 & .168 \\
\hline \multicolumn{6}{|c|}{$\begin{array}{l}\text { Nota: } \mathrm{R}^{2}=0.118 \text { para el paso } 1(p<0.001), \mathrm{R}^{2}=0.174 \text { para el paso } 2(p \\
<0.001), \mathrm{R}^{2}=0.193 \text { para el paso } 3(p<0.05), \mathrm{R}^{2}=0.210 \text { para el paso } \\
4(p<0.05) .\end{array}$} \\
\hline
\end{tabular}

En la tabla 9 se muestran los antecedentes predictivos de la actualización social; en el primer paso se observa la participación política comunitaria, explicando $7.5 \%$ de la varianza; en el segundo paso está la violencia física y daño a la propiedad privada como predictor negativo, explicando $9 \%$ de la varianza, lo que indica un modelo predictivo de eficacia baja.

Tabla 9.

Resumen del análisis de regresión paso a paso para los predictores de actualización social

\begin{tabular}{l|l|l|l|l|l|}
\multicolumn{1}{|c}{ VARIABLES } & \multicolumn{5}{c}{$\begin{array}{c}\text { M INTERVALO DE } \\
\text { CONFIANZA }\end{array}$} \\
\hline $\begin{array}{l}\text { 1er Paso } \\
\text { Interés } \\
\text { político }\end{array}$ & .154 & .031 & .293 & .093 & .215 \\
\hline $\begin{array}{l}2^{\circ} \text { Paso } \\
\text { Autoritarismo } \\
\text { de derecha }\end{array}$ & -.113 & .054 & -.123 & -.219 & -.007 \\
\hline $\begin{array}{l}\text { Nota: } \mathrm{R}^{2}=0.075 \text { para el paso } 1(p<0.001), \mathrm{R}^{2}=0.090 \text { para el paso } 2(p \\
<0.05) .\end{array}$ \\
\hline
\end{tabular}

En la tabla 10 se muestran los antecedentes predictivos de la coherencia social; en el primer paso se localiza la eficacia política global, explicando $8 \%$ de la varianza; en el segundo paso se incorpora la participación política comunitaria, explicando $10.8 \%$ de la varianza, lo cual indica un modelo predictivo de eficacia baja.

\section{Tabla 10.}

Resumen del análisis de regresión paso a paso para los predictores de coherencia social

\begin{tabular}{|c|c|c|c|c|c|}
\hline \multirow{2}{*}{ VARIABLES } & \multirow[t]{2}{*}{ B } & \multirow{2}{*}{ EE } & \multirow[t]{2}{*}{ B } & \multicolumn{2}{|c|}{$\begin{array}{l}\text { INTERVALO DE } \\
\text { CONFIANZA }\end{array}$} \\
\hline & & & & Mínimo & MÁxIMo \\
\hline $\begin{array}{l}\text { 1er Paso } \\
\text { Interés } \\
\text { político }\end{array}$ & .193 & .058 & 209 & .079 & .307 \\
\hline $\begin{array}{l}2^{\circ} \text { Paso } \\
\text { Autoritarismo } \\
\text { de derecha }\end{array}$ & .122 & .041 & .184 & .040 & 204 \\
\hline
\end{tabular}

\section{Discusión}

Los resultados correlaciones respecto a la relación entre el IP y las subescalas que conforman el BS son similares a los encontrados por Laca-Arocena et al. (2010) y Laca-Arocena et al. (2011); mantienen una relación positiva, débil y significativa entre todas las subescalas, excepto para AS e IP, donde la relación tiende a cero $(r$ $=0.035$; tabla 1 ). 
En la investigación desarrollada por Coppari et al. (2013), ya mencionada, se encontró una relación positiva y significativa entre la PP y el BS; esta correlación fue tan alta que podría indicar la evaluación de un mismo constructo; sin embargo, los resultados de esta investigación indican que la PP y el BS son constructos diferenciables. Las discrepancias de resultados tal vez se deben a las diferencias de muestras; en el estudio de Coppari et al. (2013) se evalúa la PP y el BS en una población rural, mientras que los participantes de este estudio eran en su mayoría universitarios de un entorno urbano. Como se muestra en la tabla 2 , hay un porcentaje de varianza explicada (2.9\%) que puede ser predicho por la eficacia política global, lo cual indica que las personas que se perciben capaces de intervenir de manera eficiente en asuntos políticos tienen una mayor probabilidad de votar.

En la tabla 3 se indican los predictores de la PP comunitaria; el interés político es la primera variable que se incorpora al modelo; esto indica que las personas interesadas en consumir información política tendrán mayor probabilidad de desarrollar conductas enfocadas a influir el curso político de su comunidad; este resultado es similar a la relación encontrada por Klesner (2007), mientras que las personas que manifiestan creencias propias del AD disminuyen su probabilidad de interactuar en cuestiones relacionadas con la PP comunitaria. Además, si las personas perciben aceptación y actualización social es más probable que interfieran en los asuntos propios de la PP comunitaria, al igual que si perciben que son capaces de influir en los procesos políticos mediante su percepción de EPG; este último resultado predictivo de la EPG sobre la PP comunitaria es congruente con lo indicado por Somuano (2005): "es claro que aquellos individuos que sienten que su comportamiento puede hacer una diferencia tengan mayores probabilidades de participar que aquellos que sienten lo contrario" (p. 70).

Respecto a la desobediencia civil no violenta (tabla 4), ésta se predice con dos variables: el IP, lo cual indica que las personas interesadas en consumir información política también estarán más dispuestas a efectuar actos de DCNV; de manera contraria, si las personas tienen creencias ideológicas de AD tendrán menos probabilidad de llevar a cabo conductas de DCNV, como manifestaciones o bloqueos de calles, u ocupación de edificios. En cuanto a la relación predictiva que el IP ejerció en los actos de DCNV, Somuano (2005) menciona que las personas interesadas en asuntos políticos tienen mayor probabilidad de dirigir su conducta hacia la realización de actos políticos, mientras que Marien, Hooghe y Quintelier (2010) encuentran datos empíricos que respaldan esta relación.
En cuanto a la VFDPP (tabla 5), se predice de manera positiva por el IP; esto indica que las personas con conocimiento político podrían estar más dispuestas a efectuar conductas violentas para conseguir sus demandas políticas en caso de no ser atendidas por la vía legal. Como predictor negativo de la VFDPP, surgió la contribución social, lo que indica que las personas que se perciben como más capaces para otorgar algo valioso a la sociedad mediante sus comportamientos tienen menor probabilidad de desplegar conductas violentas para que sus demandas políticas sean atendidas; también señala que la baja contribución social puede relacionarse con aumentos en la frustración que desembocan en VFDPP (Somuano, 2005).

Comenzando con las variables de BS, el predictor de la IS es la EPG (tabla 6), lo cual indica que las personas que creen ser capaces de intervenir de manera efectiva en los asuntos políticos tienen mayor probabilidad de experimentar creencias y sentimientos de que son valorados en su comunidad. Por otro lado, el predictor de la AS es la PPC (tabla 7), lo cual advierte que las personas que participan en actividades políticas de orden comunitario tienen más probabilidad de desarrollar una percepción positiva de las personas que interactúan en su entorno.

La variable de CS (tabla 8) es predicha en primera instancia por la EPG; estos resultados sugieren que las personas que creen ser capaces de intervenir de manera efectiva en los procesos políticos son más propensas a generar creencias de que son capaces de hacer aportaciones valiosas a la sociedad mediante su trabajo. En segundo término, están las creencias propias del AD como predictor negativo, lo cual demuestra que las personas que favorecen los sistemas estatales rígidos son propensas a experimentar una disminución en su percepción de las aportaciones que pueden hacer a la sociedad. En tercer término se incorpora como predictor negativo la VFDPP; de este resultado se infiere que las personas más propensas a realizar actos violentos para conseguir sus demandas políticas, también son más propensas a experimentar una disminución en su capacidad de aportación social. En cuarto término surge la PPC; de esto se deduce que las personas que se alistan en actividades políticas para resolver problemas de su comunidad aumentan la probabilidad de experimentar que son capaces de hacer aportaciones sociales relevantes (Keyes y Shapiro, 2004).

En la tabla 9 están los predictores de la ACTS; el primero es la PPC, lo cual indica que las personas que desarrollan actividades políticas relacionadas con su comunidad tienen mayor probabilidad de generar una percepción de que existe progreso y desarrollo social. El segundo predictor es negativo (VFDPP); esto sugiere que las personas capaces de efectuar actos violentos para 
legitimar sus objetivos políticos tienen menor probabilidad de generar una creencia orientada a confiar en el gobierno, el progreso y el desarrollo social.

Por último, los predictores de la COHS (tabla 10) indican que las personas con mayor EPG son más propensas a creer que es posible entender lo que sucede en el mundo; además, las personas que participan en actividades propias de la PPC tienen mayor probabilidad de generar una creencia de que son capaces de entender los distintos fenómenos sociales que acontecen en el mundo.

Respecto a los resultados mencionados de las variables antecedentes con valor predictivo para las subescalas de BS, son contrarios a lo encontrado por Laca-Arocena et al. (2010); en esta investigación, el IP no fue un predictor significativo para las variables que componen el BS; sin embargo, en el trabajo de Laca-Arocena et al. (2010) se encontró que el IP fue un predictor significativo de fuerza débil para las variables de aceptación, contribución y coherencia social.

\section{Conclusiones}

Al sintetizar los resultados obtenidos se puede contestar satisfactoriamente la pregunta de investigación, así como el logro de los objetivos al observar un modelo predictivo donde la participación política se predice por las siguientes variables: eficacia política global, interés político, autoritarismo de derecha, aceptación social, actualización social y contribución social; mientras que el bienestar social se predice por los siguientes antecedentes: eficacia política global, participación política comunitaria, autoritarismo de derecha, violencia física y daño a la propiedad privada.

La contribución de encontrar una relación positiva entre el bienestar y participación política junto con investigaciones previas (Laca-Arocena et al., 2010; Laca-Arocena et al., 2011; Coppari et al., 2013), sustenta la relación entre dos procesos sociales que se presentan en la vida de las personas que desencadena una serie sentimientos, emociones, un pensamiento de utilidad y contribución a la sociedad, además de significar tanto un bienestar individual como existencial.

Es necesario puntualizar que los resultados son matizados por una población joven y universitaria donde se entredice que mayor nivel educativo favorece la participación política, aunque con resultados de Murga (2009) el tema, y en específico la relación de jóvenes y su involucramiento en la política, es inconcluso. El objetivo de la investigación no fue demostrar la participación política de los jóvenes; sin embargo, los datos muestran predicciones relevantes para que se propicien conductas de participación política.

$\mathrm{Al}$ destacar que la única variable antecedente con valor significativo para predecir la conducta de voto es la eficacia política global ( $2.9 \%$ de varianza), se refleja que la conducta del voto es fenómeno que debe ser investigado porque es la manera más común de participación; es difícil de predecir.

Es prudente mencionar la necesidad de hacer más estudios para evaluar el BS con participantes mexicanos porque, como mencionan Keyes y Shapiro (2004, p. 354), "en la población estadounidense literalmente no hay estudios de bienestar social"; en México, el estado respecto a la investigación de este constructo es escaso. Asimismo, el análisis de fenómenos políticos desde la Psicología permite establecer un debate teórico acerca de las explicaciones que hay en la política, además de promover metodologías donde se deba presentar la integración de otras disciplinas. Por otro lado, el involucramiento de la Psicología en temas como el bienestar social significa revalorar la función de los profesionales ante su obligación con la sociedad y cómo dar respuestas científicamente validadas; así, este trabajo no sólo tenía la enmienda de buscar un modelo predictivo, sino también una reflexión de los alcances de la disciplina en temas políticos, donde se pude aportar un valioso conocimiento en pro de un bienestar.

Por último, se mencionan algunas debilidades de esta investigación; su diseño transversal nos impide generar conclusiones de orden causal, al ser los participantes estudiantes universitarios en su mayoría o personas con un antecedente educativo de licenciatura, es difícil que los resultados de esta investigación puedan generalizarse para otros contextos poblacionales; el uso de cuestionarios de autorreporte también conlleva una serie dificultades, como la deseabilidad social que puede afectar la evaluación de variables como la conducta de votar (Tourangeau y Yan, 2007).

\section{Referencias}

Argibay, J. C. (2006). Técnicas psicométricas. Cuestiones de validez y confiabilidad. Subjetividad y procesos cognitivos, (8), 15-33.

Arias-Cardona, A. \& Alvarado, S. (2015). Jóvenes y política: de la participación formal a la movilización informal. Revista Latinoamericana de Ciencias Sociales, Niñez y Juventud 13(2), 581-594.

Blanco, A. \& Díaz, D. (2005). El bienestar social: su concepto y medición. Psicothema, 17(4), 582-589.

Brussino, S., Medrano, L., Sorribas, P. \& Rabbia, H. H. (2011). Young adults' knowledge of politics: Evaluating the role of 
socio-cognitive variables using structural equations. The Spanish Journal of Psychology, 14(01), 183-194.

Brussino, S., Rabbia, H. H. \& Sorribas, P. (2009). Perfiles sociocognitivos de la participación política de los jóvenes. Interamerican Journal of Psychology, 43(2), 279-287.

Cárdenas, V. G. (2011). Capital Social y bienestar social. En Uribe, F. J. y Acosta M. T. (eds.), Bienestar social y democracia (pp. 19-48). México, D. F.: Miguel Ángel Porrúa.

Coppari, N., Aponte, A., Ayala, P., Moreno, M., Quevedo, C., Sola, M. \& Velázquez, T. (2013). Percepción de bienestar social y participación política en adultos de una comunidad rural en Minga Guazú, Alto Paraná. Eureka (Asunción) en Línea, 10(1), 55-67.

Córdova, L., Alejandre, O. \& Vázquez del Mercado, S. (2015). El déficit de la democracia en México Encuesta Nacional de Cultura Política. Universidad Autónoma de México, México.

Delfino, G., Zubieta, E. \& Muratori, M. (2010). Participaciòn política: conceptos y modalidades. Anuario de Investigaciòn 17, 211-220.

Delfino, G., Zubieta, E. \& Muratori, M. (2013). Tipos de participación política: análisis factorial confirmatorio con estudiantes de Argentina. Psicología Política, 13(27), 301318.

Diener, E., Suh, E. M., Lucas, R. E. \& Smith, H. L. (1999). Subjective well-being: Three decades of progress. Psychological bulletin, 125(2), 276-302.

García y Barragan, F. (2008). Valoración de una Escala de Participación Política. Revista de Psicología Social y Personalidad. Recuperado de http://ugto.academia.edu/ LuisGarcia/Papers

Hayes, A. F. (2013). Multiple Linear Regression. Author (ed.), Introduction to Mediation, Moderation, and Conditional Process Analysis. A Regression-Based Approach (pp. 5982). New York: The Guilford Press.

Instituto Federal Electoral [IFE] (2013). Estudio Censal de la Participación Ciudadana en las Elecciones Federales de 2012. Recuperado de: http://www.ine. $\mathrm{mx/docs/}$ IFE-v2/DECEYEC/DECEYEC-Estudios/nvestigaciones/ InvestigacionIFE/Estudio_Censal_Participacion Ciudadana_2012.pdf

Keyes, C. L. M. (1998). Social well-being. Social Psychology Quarterly, 121-140.

Keyes, C. L. \& Shapiro, A. D. (2004). Social well-being in the United States: A descriptive epidemiology. How healthy are we, 350-372.

Klesner, J. L. (2007). Social capital and political participation in Latin America: evidence from Argentina, Chile, Mexico, and Peru. Latin American Research Review, 1-32.

Laca-Arocena, F. A., Mejía-Ceballos, J. C. \& Yañez-Velasco, C. (2010). Identidad mexicana e interés político: Predictores de bienestar social y anomia. Acta Universitaria, 20(2), 40-49.

Laca-Arocena, F. A., Santana-Aguilar, H., Ochoa-Madrigal, Y. \& Mejía-Ceballos, J. C. (2011). Percepciones de bienestar social, anomia, interés e impotencia política en relación con las actitudes hacia la democracia. Liberabit, 17(1), 7-18.

Leyva, O., Muñiz, C. \& Flores, M. (2016). La conformación de actitudes políticas de los jóvenes universitarios en el contexto preelectoral 2015 en Nuevo León. Revista
Mexicana de Opinión Pública (21), 51-70.

Marien, S., Hooghe, M. \& Quintelier, E. (2010). Inequalities in noninstitutionalised forms of political participation: A multi-level analysis of 25 countries. Political Studies, 58(1), 187-213.

Moreno, E. (2008). Análisis de correlación y regresión. Autor (ed.), Manual de uso de SPSS (pp. 165-203) Madrid: UNED IUED Instituto Universitario de Educación a Distancia.

Murga, A. (2009). La participación política de los estudiantes universitarios en el primer gobierno de alternancia en México. Religión y Sociedad 2(45), 45-63.

Niemi, R. G., Craig, S. C. \& Mattei, F. (1991). Measuring internal political efficacy in the 1988 National Election Study. American Political Science Review, 85(04), 1407-1413.

Rodríguez, G. (2004). Significado de la participación política en habitantes del valle de México. Psicología Política, (29), 68-78.

Rollero, C. (2013). Testing predictors of social well-being: are they the same for men and women?. Psychology of WellBeing, 57-64.

Ryff, C. D. \& Keyes, C. L. M. (1995). The structure of psychological well-being revisited. Journal of Personality and Social Psychology, 69(4), 719-727.

Salanova, M., Llorens, S. \& Schaufeli, W. B. (2011). "Yes, I can, I feel good, and I just do it!" On gain cycles and spirals of efficacy beliefs, affect, and engagement. Applied Psychology, 60(2), 255-285.

Somuano, M. F. (2005). Más allá del voto: modos de participación política no electoral en México. Foro Internacional, 65-88.

Son, J. \& Wilson, J. (2012, September). Volunteer Work and Hedonic, Eudemonic, and Social Well-Being. In Sociological Forum (Vol. 27, No. 3, pp. 658-681). Blackwell Publishing Ltd.

Sorribas, P. \& Brussino, S. (2017). Participación política: el aporte discriminante de actitudes ideológicas, valores y variables sociopsicológicas. Revista de Psicología, 35 (1), 311-345.

Temkin, B. \& Flores-Ivich, G. (2017). Tipos de participación política y bienestar subjetivo: un estudio mundial. Estudios Sociológicos 35(104), 319-341.

Tourangeau, R. \& Yan, T. (2007). Sensitive questions in surveys. Psychological Bulletin, 133(5), 859-883.

Várela,E., Loreto, M. \& Cumsille, P. (2015). ¿Es la participación política convencional un indicador del compromiso cívico de los jóvenes?. Universitas Psychologica, 14(2), 731- 746.

Velasco, P. W. (2015). Una aproximación bio-psico-socio-cultural al estudio del bienestar subjetivo en México: Un modelo explicativo-predictivo (Tesis doctoral). Recuperada de http://132.248.9.195/ptd2015/junio/406012454/Index.html

Weber, C. \& Federico, C. M. (2007). Interpersonal attachment and patterns of ideological belief. Political Psychology, 28(4), 389-416.

World Health Organization [Organización Mundial de la Salud] (1998). Health promotion glossary. Recuperado de http:// apps.who.int/iris/bitstream/10665/64546/1/WHO_HPR_ HEP 98.1.pdf

Zubieta, E. M. \& Delfino, G. (2010). Satisfacción con la vida, bienestar psicológico y bienestar social en estudiantes universitarios de Buenos Aires. Anuario de investigaciones, $17,277-283$. 
Artículo Empírico | Participación política y bienestar social... Chavéz-ortega eZ Valdez-Estrella.

\section{Meta-Análisis del Artículo}




\section{Dimensión Cuantitativa}

\section{Perfil de Evaluación entre pares}
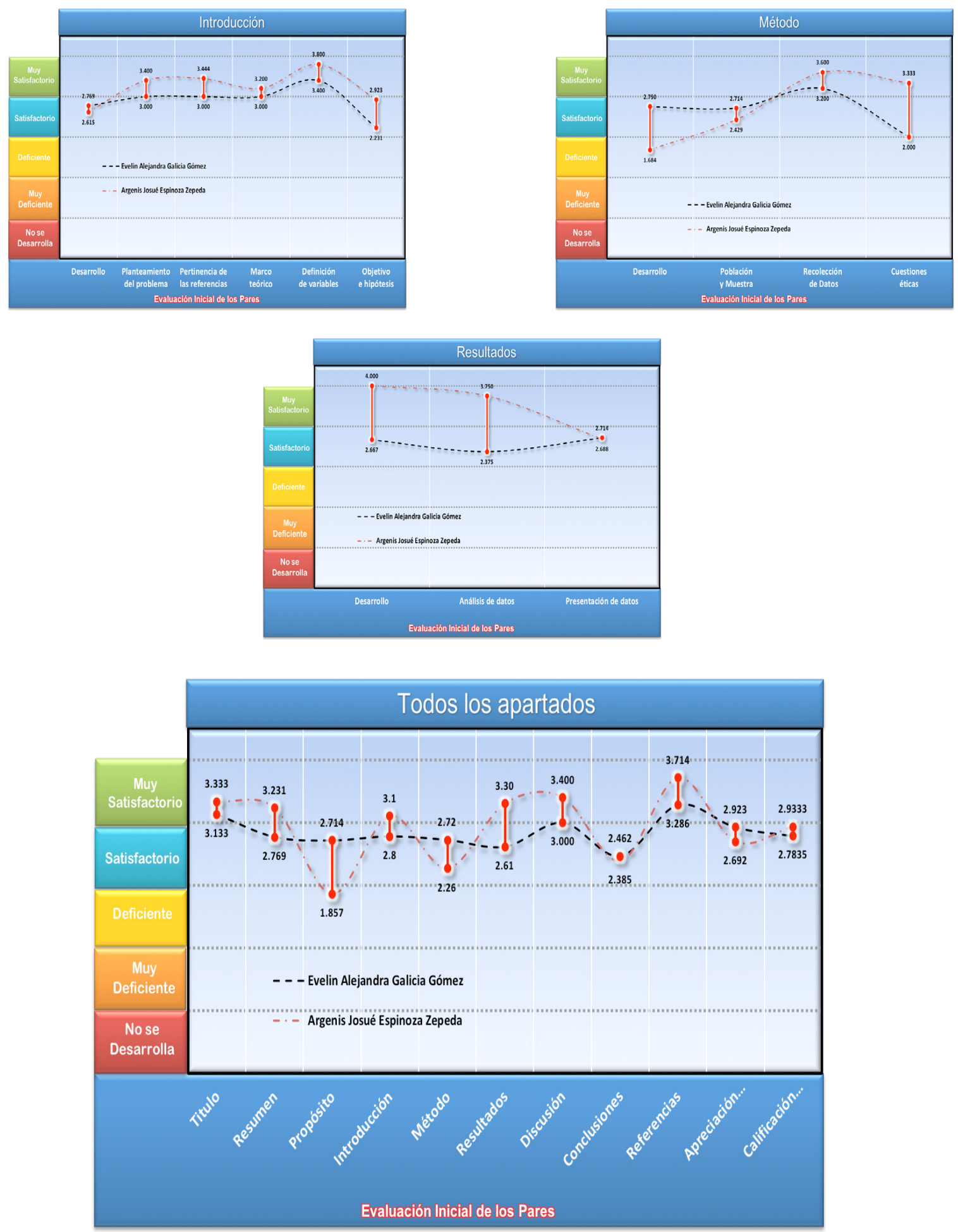


\section{Índice de Concordancia}

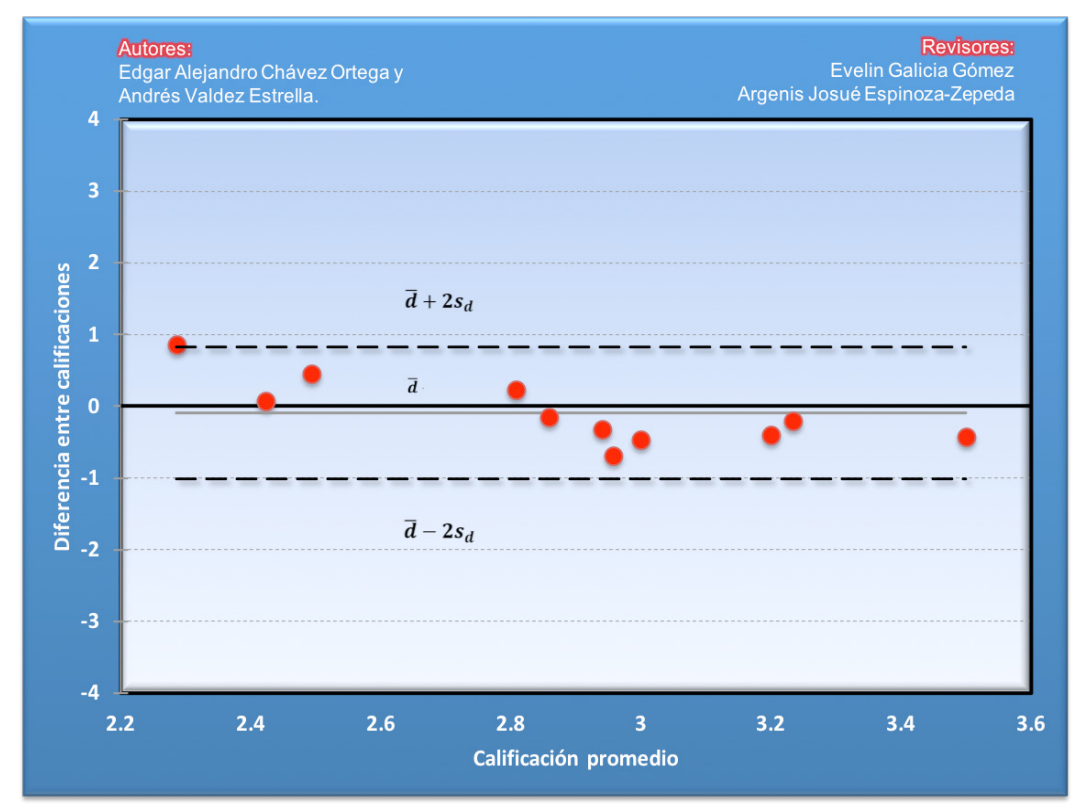

Índice de Acuerdo

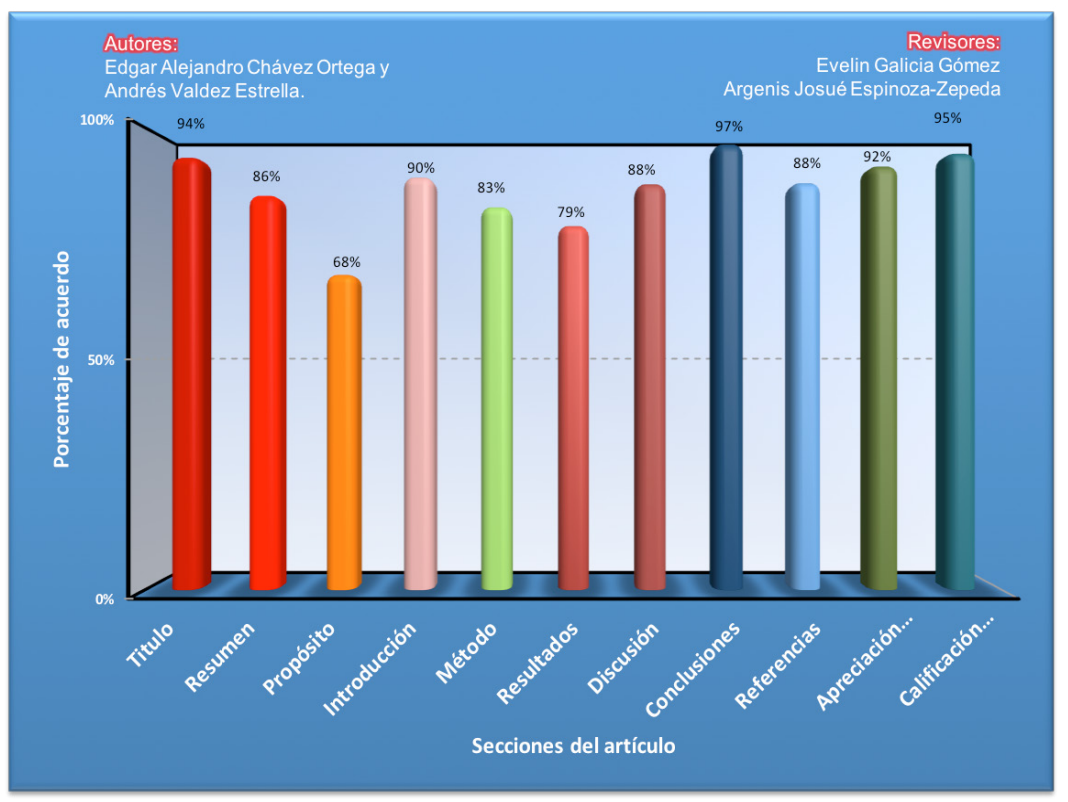




\begin{tabular}{|c|c|}
\hline Revisor 1 & Revisor 2 \\
\hline Evelin Galicia Gómez & Argenis Josué Espinoza Zepeda \\
\hline \multicolumn{2}{|c|}{ Título/Autoría } \\
\hline $\begin{array}{l}\text { El título del manuscrito a revisión cuenta con los ele- } \\
\text { mentos fundamentales, sin embargo, puede ser más } \\
\text { explícito al incluir que el modelo es aplicable a cierta } \\
\text { población, por ejemplo; "La participación política y } \\
\text { el bienestar social, un modelo predictivo en jóvenes } \\
\text { mexicanos" }\end{array}$ & $\begin{array}{l}\text { Participación política y bienestar social; un modelo pre- } \\
\text { dictivo con jóvenes mexicanos }\end{array}$ \\
\hline \multicolumn{2}{|l|}{$\begin{array}{ll} & \text { Resumen } \\
\end{array}$} \\
\hline $\begin{array}{l}\text { El resumen es conciso pero no integra datos obtenidos } \\
\text { de la investigación ni presenta cifras o porcentajes, no } \\
\text { cumple con el criterio de máximo } 150 \text { palabras, al com- } \\
\text { parar investigaciones anteriores tampoco aparecen da- } \\
\text { tos que nos den una idea del resultado obtenido. }\end{array}$ & $\begin{array}{l}\text { No se agregaron resultados en con valores numéricos. los } \\
\text { cuales están bien desarrollados y pueden ser retomados de } \\
\text { la sección de resultados del cuerpo del artículo. }\end{array}$ \\
\hline \multicolumn{2}{|c|}{ Próposito del Estudio } \\
\hline $\begin{array}{l}\text { No se encuentra como tal una pregunta de investiga- } \\
\text { ción por lo cual se "deduce" cuales son las interrogantes } \\
\text { que pueden surgir, la sugerencias sería que a manera de } \\
\text { esclarecer el propósito de la investigación se realizaran } \\
\text { ñas preguntas que servirán de guía para establecer si el } \\
\text { método utilizado es el correcto y si el estudio responde } \\
\text { a las interrogantes hechas. }\end{array}$ & $\begin{array}{l}\text { En el marco teórico no está redactada la justificación de } \\
\text { la investigación. Además pese a que en el resumen esta } \\
\text { claramente descrito el objetivo, éste no es mencionado } \\
\text { a lo largo del artículo, por lo que se recomienda agregar } \\
\text { dichos puntos fundamentales faltantes. }\end{array}$ \\
\hline \multicolumn{2}{|c|}{ Introducción } \\
\hline $\begin{array}{l}\text { No se encuentra como tal una pregunta de investiga- } \\
\text { ción por lo cual se "deduce" cuales son las interrogantes } \\
\text { que pueden surgir, la sugerencias sería que a manera de } \\
\text { esclarecer el propósito de la investigación se realizaran } \\
\text { ñas preguntas que servirán de guía para establecer si el } \\
\text { método utilizado es el correcto y si el estudio responde } \\
\text { a las interrogantes hechas. }\end{array}$ & $\begin{array}{l}\text { Como ya se mencionó, hace falta se desarrolle en el ar- } \\
\text { tículo el porqué de la investigación, ya que no existe } \\
\text { justificación. }\end{array}$ \\
\hline
\end{tabular}




\begin{tabular}{|c|c|}
\hline Revisor 1 & Revisor 2 \\
\hline \multicolumn{2}{|c|}{ Método } \\
\hline $\begin{array}{l}\text { En una investigación es muy importante que se considere } \\
\text { os aspectos éticos, los cuales deben ser tratados con sumo } \\
\text { cuidado, para segurar la confidencialidad de los partici- } \\
\text { pantes, se debe tener una prueba de que el estudio que } \\
\text { se realiza es consensuado. En la metodología se debe ser } \\
\text { muy específicos, sobre todo puntualizar el cómo y el por } \\
\text { qué, se elige cierta población para el estudio y no olvidar } \\
\text { los criterios de inclusión y exclusión dentro del estudio. }\end{array}$ & $\begin{array}{l}\text { En la sección de materiales de debe poner lo que en el ar- } \\
\text { tículo está definido como "Mediciones". Ahora bien, con } \\
\text { respecto a la población objetivo, no se hace mención del } \\
\text { porqué esa población. }\end{array}$ \\
\hline
\end{tabular}

\section{Resultados}

Al no existir una pregunta de investigación clara, solo se puede deducir el resultado, de acuerdo a lo que expone el título, que en su caso no sustituye a la pregunta de investigación, or lo cual no se puede considerar como pregunta de investigación.

Se recomienda agregar gráficos al trabajo.

\section{Discusión}

La discusión de los resultados obtenidos lleva un orden cronológico de acuerdo a los resultados obtenidos, pero sin una pregunta de investigación establecida solo se puede hacer un comparativo entre esta investigación

Solo mejorar la redacción, al igual que en el resto de las secciones del artículo.

y las anteriores lo cual no responde al por qué se decidió hacer este estudio.

\section{Conclusiones}

Aunque las conclusiones tienen una estructura adecuada, faltó relacionarlas con los resultados obtenidos, mencionar hacia dónde se dirige la siguiente investigación y si habrá otra, es importante que en este estado se considere las contribuciones que hará este estudio y la relevancia que tendrá en el futuro.

En la conclusión no se hizo referencia a si se cumplió o no el objetivo de la investigación, cuando es fundamental mencionarlo antes de proponer futuras investigaciones.

\section{Referencias}

Los criterios para realizar este apartado se apegan a Manual de Estilo de Publicaciones de la APA

Verificar en el manual de estilo el uso de la sangría en referencias. 


\section{Historia del Proceso}

\section{EDITORIAL}

